\title{
EOPs: Manejo de la epistaxis idiopática recurrente en niños
}

\author{
Management of recurrent idiopatic epistaxis in children
}

Milthon Rodríguez $\mathrm{R}^{*}$ y Federico Augustovski**

\begin{abstract}
20. Resumen
A partir del caso clínico de una niña con episodios de epistaxis recurrente surgió la duda sobre la efectividad de las intervenciones médicas existentes para disminuir la frecuencia de los episodios de epistaxis en niños con esta condición. Se formuló la pregunta clínica con formato PICO*, y se describieron los principales estudios hallados. Se encontró evidencia que sugiere no haber diferencia entre administrar o no tratamiento en esta población (crema antiséptica, vaselina o cauterización con nitrato de plata) tanto a corto como largo plazo.

\section{Abstract}

From the case of a child with recurrent episodes of epistaxis, doubt arose about the effectiveness of existing medical interventions to decrease the frequency of episodes of epistaxis in children with this condition. A question was formulated with the $\mathrm{PICO}^{*}$ format and the main studies were described. We found evidence that suggest no difference between giving or not treatment (antiseptic cream, petroleum jelly, or cauterization with silver nitrate) both short and long term in this population.
\end{abstract}

Palabras clave: epistaxis recurrente, efectividad, tratamiento. Key words: recurrent nosebleed, effectiveness, treatment.

Rodriguez M, Augustovski F. Manejo de la epistaxis idiopática recurrente en niños. Evid Act Pract Ambul 13(4). 158-159 Oct-Dic 2010

\section{Escenario Clínico}

Un médico de familia atiende a una niña de cinco años sin antecedentes médicos conocidos, la cual ha sido llevada al consultorio en repetidas ocasiones por episodios espontáneos y autolimitados de epistaxis. Estos no tienen asociación aparente a traumatismos ni presentan repercusión hemodinámica, y aparecen por momentos asociados al calor $\mathrm{o}$ a la actividad física. Al examen físico no se encuentra ningún hallazgo relevante en el área nasal ni en el resto del cuerpo y mucosas. Mediante análisis de laboratorios practicados a la niña, se descartaron alteraciones hematológicas de base.

\section{Pregunta clínica que generó el caso}

¿En niños y adolescentes con episodios de epistaxis recurrente (Población y Problema), existe alguna intervención médica (Intervención) que haya demostrado ser mejor que la no intervención (Comparación) para disminuir la frecuencia de dichos episodios (Outcomes o resultados)?

\section{Estrategia y resultados de la búsqueda}

Se realizó una búsqueda en PubMed, The Cochrane Library, LILACS, DARE (Database of Abstracts of Reviews of Effects) y HTA (Health Technology Assessment) utilizando como palabras claves: "epistaxis", "recurrent epistaxis", "nosebleeds" AND "children"; y limitando la búsqueda a pacientes de 0 a 18 años, a ensayos clínicos aleatorizados, revisiones sistemáticas y metanálisis, durante un periodo de publicación no mayor a los diez años previos a la búsqueda y en los idiomas español e inglés.

La búsqueda arrojó dos revisiones sistemáticas publicadas en 2008, una de ellas de la Colaboración Cochrane ${ }^{1}$ que incluyó publicaciones hasta agosto de 2003 y otra de la revista Clinical Evidence $^{2}$ (del grupo BMJ) cuya búsqueda fue inclusiva hasta abril de 2008. Ambas evaluaron tres ensayos aleatorizados sobre intervenciones para el manejo de la epistaxis recurrente en niños. Para detectar potenciales publicaciones posteriores, se realizó una búsqueda a partir de 2008 en las fuentes previamente indicadas, encontrándose una publicación de 2008 que daba seguimiento a pacientes de uno de los estudios ya evaluados en las dos revisiones sistemáticas previamente mencionadas ${ }^{3}$, y otro estudio, con fecha de publicación de 2009, que fue excluido por requerir de sus participantes la presencia de un vaso sangrante visible en el tabique nasal como criterio de inclusión.

\section{Algunas consideraciones sobre la epistaxis recurrente}

Se denomina epistaxis recurrente al sangrado nasal autolimitado $\sin$ una etiología claramente definida ${ }^{2}$.

Constituye una causa frecuente de consulta en atención primaria y se ha visto que por lo menos el $9 \%$ de los niños y adolescentes presentan episodios frecuentes de epistaxis autolimitados, ${ }^{4}$ pero pese a ello no hay consenso en cuanto a las definiciones de frecuencia y severidad de las recurrencias.

La gran mayoría de los episodios (hasta un $90 \%)^{5}$ se originan en el área de Little, en la región anterior del tabique nasal donde se forma el plexo de Kiesselbach debajo de la mucosa nasal ${ }^{6}$, y son más comunes durante el invierno ${ }^{7}$ cuando abundan las infecciones del tracto respiratorio superior y disminuye la humedad ambiental ${ }^{8}$. También es frecuente su aparición en climas cálidos y secos con poca humedad'. Son raros los episodios antes de los dos años y después de los 144

La causa de la epistaxis recurrente en la niñez se ha atribuido principalmente a la formación de costras, al antecedente de vestibulitis nasal, sequedad de mucosas, trauma digital local (rascado de nariz) ${ }^{9}$ e incluso a la colonización nasal con Staphylococcus aureus ${ }^{10}$; aunque en la mayor parte de los casos no puede establecerse ninguna causa directa ${ }^{9}$.

En vista de lo anterior, se han sugerido múltiples opciones de tratamiento para intentar disminuir la recurrencia de la epistaxis, las cuales van desde la aplicación tópica de cremas antisépticas o humectantes hasta la cauterización del área sospechosa de hemorragia, con resultados variables.

\section{Resumen de la Evidencia}

Burton MJ, Dorée CJ. Intervenciones para la epistaxis (hemorragia nasal) idiopática recurrente en niños (Revisión Cochrane traducida). En: La Biblioteca Cochrane Plus, 2008 Número 2.

Se trata de una revisión sistemática (no pudo realizarse un metanálisis debido a la heterogeneidad de los datos) que evaluó tres ensayos clínicos aleatorizados de intervenciones propuestas para el manejo de la epistaxis idiopática recurrente en niños. Las características y principales resultados de los estudios se reflejan en la tabla 1.

\footnotetext{
* Residencia de Medicina Familiar, Caja de Seguro Social y Universidad de Panamá. milthonrr@yahoo.com

** Servicio de Medicina Familiar y Comunitaria del Hospital Italiano de Buenos Aires. Instituto de Efectividad Clínica y Sanitaria. federico.augustovski@hospitalitaliano.org.ar
} 
Tabla 1: Efectividad de diferentes estrategias para la prevención de la epistaxis recurrente.

\begin{tabular}{|c|c|c|c|c|c|c|c|}
\hline \multirow[t]{2}{*}{ Estudio } & \multirow[t]{2}{*}{ Población } & \multirow[t]{2}{*}{$\begin{array}{l}\text { Intervenciones (tipo de } \\
\text { estrategia) }\end{array}$} & \multicolumn{2}{|c|}{$\begin{array}{l}\text { \% de pacientes con } \\
\text { epistaxis recurrente }\end{array}$} & \multirow[t]{2}{*}{$\mathrm{p} / \mathrm{NNT}$} & \multirow[t]{2}{*}{ GRADE* } & \multirow[t]{2}{*}{ Comentarios } \\
\hline & & & Estrategia 1 & Estrategia 2 & & & \\
\hline Kubba (2001) & $\begin{array}{l}103 \text { pacientes } \\
\text { de } 3 \text { a } 13 \text { años }\end{array}$ & $\begin{array}{l}\text { Naseptin }{ }^{\circ}(1) \text { contra no } \\
\text { tratamiento (2) }\end{array}$ & \begin{tabular}{l|}
45 \\
41
\end{tabular} & $\begin{array}{l}71 \\
56\end{array}$ & $\begin{array}{l}0,01 / 4 \\
0,14 /-\end{array}$ & Baja & $\begin{array}{l}\text { Pérdida de seguimiento de } 14,5 \% \text {. Simple ciego*. } \\
\text { Análisis por protocolo* }\end{array}$ \\
\hline Laughran (2003) & $\begin{array}{l}105 \text { pacientes de } \\
\text { entre } 1 \text { y } 14 \text { años }\end{array}$ & $\begin{array}{l}\text { Vaselina }{ }^{\beta}(1) \text { contra no } \\
\text { tratamiento (2) }\end{array}$ & 73 & 66 & $0,47 /-$ & $\begin{array}{l}\text { Moderada } \\
\text { Moderada }\end{array}$ & \begin{tabular}{|l} 
Análisis por intención de tratar* \\
Mejor diseño metodológico de los tres estudios
\end{tabular} \\
\hline Ruddy (1991) & 48 pacientes & $\begin{array}{l}\text { Naseptin₹ (1) contra cauterización } \\
\text { con nitrato de plata (2) }\end{array}$ & 45 & 38 & $0,56 /-$ & & $\begin{array}{l}\text { No quedó claro el modo de ceguera* ni la } \\
\text { distribución al azar de los participantes }\end{array}$ \\
\hline
\end{tabular}

' Naseptin crema (Neomicina 0,5\% y Clorhexidina 0,1\%), que se topicó en el tabique nasal dos veces al día por cuatro semanas, evaluándose la respuesta a tratamiento al cabo de otras cuatro. ${ }^{\beta}$ La vaselina se topicó en el tabique nasal dos veces al día por cuatro semanas, evaluándose la respuesta a tratamien-

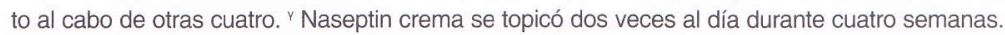

Valoración GRADE. Evidencia Baja: indica que resulta muy probable que la investigación adicional tenga un impacto importante en nuestra confianza sobre la estimación del efecto y que probablemente modifique dicha estimación. Evidencia Moderada: indica que resulta probable que la investigación adicional tenga un impacto importante en nuestra confianza sobre la estimación del efecto y que probablemente modifique dicha estimación.

\section{McGarry G. Nosebleeds in children. Clinical Evidence 2008;05:311}

Esta revisión narrativa también incluyó los tres ensayos analizados en la Revisión Cochrane antes detallada, y aplicó el sistema de valoración GRADE* para evaluar la calidad de la evidencia que sustenta a las diferentes intervenciones (ver cuadro 1).Sin embargo en esta revisión solo se tomaron en cuenta los resultados favorables del estudio de Kubba analizado por protocolo*. Esta revisión consideró además otro ensayo que evaluaba a niños y adultos (Murthy 1999) el cual no fue incluido en el presente EOPs.

Robertson S, Kubba H. Long-term effectiveness of antiseptic cream for recurrent epistaxis in childhood: five-year follow up of a randomised, controlled trial. The Joumal of Laryngology \& Otology (2008), 122, 1084-1087

Esta publicación, más reciente, buscaba evaluar mediante un diseño de cohorte retrospectiva* la efectividad a largo plazo (cinco años después) de los tratamientos empleados para el control de epistaxis en los pacientes que participaron inicialmente en el estudio de Kubba en 2001. De aquellos pacientes que completaron el estudio (88) sólo fue posible contactar a $60(68 \%)$, entre junio y setiembre de 2006 , de los cuales 39 (65\%) continuaba con episodios de epistaxis (66\% en los que se asignaron a utilizar la crema antiséptica y $64 \%$ en el grupo control sin tratamiento, $p=0,89$ ). De entre los niños que continuaron presentando epistaxis, sólo cinco $(13 \%)$ acudieron a un servicio de urgencias en donde se les realizó cauterización, junto con la aplicación de la crema antiséptica en alguna ocasión; mientras que únicamente ocho niños (20\%) fueron referidos de vuelta a la Clínica de Otorrinolaringología para continuar con el tratamiento de la epistaxis. La frecuencia de sangrado en los niños evaluados en 2006 fue menor que la del 2001, aunque el 49\% (19/39) continuó con epistaxis al menos una vez por mes. Cinco años después de terminado el primer estudio, se dividió en tres grupos a los 60 niños contactadios de acuerdo a la asignación inicial, así el 18\% (11/60) no recibió ningún tratamien- to, $30 \%(18 / 60)$ recibió únicamente la crema antiséptica (Neomicina - Clorhexidina) y 52\% (31/60) crema antiséptica junto con cauterización. En estos, continuaron con epistaxis un $45 \%$ (5/11) en el grupo $\sin$ tratamiento, 56\% (10/18) en los que sólo utilizaron crema y $77 \%(24 / 31)$ en el grupo que necesitó cauterización adicional al uso de la crema antiséptica. Cabe señalar que durante estos cinco años que siguieron al estudio, todo niño que consultó en más de una ocasión por epistaxis se le realizó cauterización en algún momento. Si bien es cierto que 28 niños (32\%) de los 88 del estudio original no pudieron ser contactados por cambio de residencia o de sus datos para este seguimiento, los autores sugieren que la muestra es representativa en virtud de ser poco probable que se afecte la frecuencia de la epistaxis por el cambio de estas circunstancias domésticas en este grupo.

* ver glosario

\section{Comentarios y Conclusiones}

De acuerdo a la literatura consultada pareciera ser que los tratamientos actuales para la disminución de la recurrencia de la epistaxis Idiopática en niños y adolescentes de hasta 15 años, no tienen mejores resultados que el no dar tratamiento activo, tanto a corto como a largo plazo.Después de cinco años de seguimiento, el $65 \%$ de los niños asignados a recibir la aplicación de crema antiséptica frente a no recibir nada, continuó con epistaxis, con un $49 \%$ de los mismos con epistaxis al menos una vez al mes; sin embargo sólo la minoría requirió acudir a la guardia o ser referido de vuelta a la Clínica de Otorrinolaringología.

Si bien hasta $2 / 3$ de los niños a cinco años de seguimiento continúan con episodios de epistaxis, la mayoría no ameritó tratamiento adicional, hecho que tal vez indique mayor comprensión de la benignidad de esta condición por parte de los padres de los niños, con menores niveles de ansiedad y mayor tolerancia, como lo señalan los autores que llevaron a cabo el estudio de seguimiento a cinco años de los niños con epistaxis ${ }^{3}$.

2. McGarry G. Nosebleeds in children. Clinical Evidence 2008:05:311 2. McGarry G. Nosebleeds in children. Clinical Evidence 2008;05:311,

Laryngology \& Otology 2008;122:1084-1087.

4. Rodeghiero F, Castaman G, Dini E. Epidemiological investigation of the prevalence
5. Ruhl TS. Epistaxis and nosebleeds. Disponible en www. essentialevidenceplus.com

6. Watkinson JC. Epistaxis. In: Kerr AG, Mackay IS, Bull TR, eds. Scott-Brown's Otolaryngology, Volume 4 Rhinology. Oxford: Butterworth-Heinemann,1997;18:1-19.

7. Pallin DJ, Chng YM, McKay MP, y col. Epidemiology of epistaxis in US emergency departments, 1992 to 2001. Ann Emerg Med. 2005;46:77-81.

8. Nunez DA, McClymont LG, Evans RA. Epistaxis: a study of the relationship with weather. Clinical Otolaryngology 1990;15:49-51.

9. Guarisco JL, Graham 3rd HD. Epistaxis in children: causes, diagnosis and treatment. Ear Nose Throat Journal 1989;68:522-38.

10. Whymark A, Crampsey D, Fraser L, y col. Childhood epistaxis and nasal colonisation with Staphyloccus aureus. Clin Otolaryngol 2007:32:230-1 